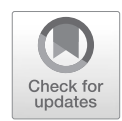

\title{
Erratum to: Numerical Models for Differential Problems
}

\author{
Alfio Quarteroni
}

Erratum to:

A. Quarteroni, Numerical Models for Differential Problems, MS\&A 16, https://doi.org/10.1007/978-3-319-49316-9

An error in the production process unfortunately led to the publication of this book before the incorporation of the corrections in the book and the Index. The version supplied here has been corrected and approved by the author.

The updated online version of this book can be found at https://doi.org/10.1007/978-3-319-49316-9 